Research Article

\title{
Nonlinear Differential Geometry Method and Its Application in Induction Motor Decoupling Control
}

\author{
Linyuan Fan ${ }^{1, *}$ and Jingyang Zhong ${ }^{2}$ \\ ${ }^{1}$ School of Statistics, Capital University of Economics and Business, Beijing 100070, China \\ ${ }^{2}$ Department of Mathematics, University of California Santa Cruz, CA 95064, U.S.A
}

Received 10 January 2015; Accepted 20 May 2016

\begin{abstract}
An alternating current induction motor is a nonlinear, multi-variable, and strong-coupled system that is difficult to control. To address this problem, a novel control strategy based on nonlinear differential geometry theory was proposed. First, a five-order affine mathematical model for an alternating current induction motor was provided. Then, the feedback linearization method was used to realize decoupling and full linearization of the system model. Moreover, a general and simplified control law was adopted to facilitate practical applications. Finally, a controller was designed using the pole assignment method. Simulation results show that the proposed method can decouple the system model into two independent subsystems, and that the closed-loop system exhibits good dynamic and static performances. The proposed decoupling control method is useful to reduce the system complexity of an induction motor and to improve its control performance, thereby providing a new and feasible dynamic decoupling control for an alternating current induction motor.
\end{abstract}

Keywords: Induction Motor; Nonlinear Differential Geometry; Decoupling Control; Full Linearization

\section{Introduction}

An induction motor (IM) has the advantages of small friction, few mechanical noise, high running speed, and low cost, among others [1]; hence, it is widely used in energy transportation, aerospace, industrial machinery, electronic communications, and many other fields, and has achieved good results [2], [3]. However, an IM is a complex multiinput multi-output (MIMO) nonlinear system with a strong coupled relationship, which makes effectively controlling rotational speed and rotor flux, as well as achieving good control performance, difficult [4], [5]. Enabling an IM to perform decoupling control is important because doing so can improve system stability and reduce the influence of noise. Therefore, realizing decoupling control and full linearization of an IM has been a focus in the field of alternating current (AC) drives.

\section{State of the art}

Numerous researchers have proposed various methods to solve the aforementioned problem; among which, the nonlinear differential geometry (NDG) method has increasingly demonstrated its superiority in decoupling and linearizing multi-variable nonlinear coupling systems [6-8]. Liao [9] conducted a comparative study of two decoupling control methods, namely, differential geometry and classical

\footnotetext{
*E-mail address: liushanyong2012@163.com ISSN: 1791-2377 (C) 2016 Eastern Macedonia and Thrace Institute of Technology. All rights reserved.
}

vector control. The results showed that vector control could only realize static decoupling, whereas differential geometry could realize full dynamic decoupling. Therefore, the differential geometry method is superior to the classical vector control method in many applications. Meng [10] introduced Kalman filtering for linear decoupling control to observe load torque change in an IM. This method could effectively track the dynamic parameters of an IM online and address the problem of input-output linearization, which could be affected by the variation parameters of the motor. The simulations showed that this technique was feasible and could achieve satisfactory results. Cao Li [11] designed a control system for a bearingless synchronous reluctance motor based on differential geometry theory and then set up the mathematical models for the radial suspension force and the torque subsystems. The simulation results showed that this control strategy could realize decoupling control and exhibited good dynamic and static performances. Yazdanpanah [12] designed a sliding mode torque and flux controller for a three-phase IM; this controller was combined with an adaptive input-output feedback linearization (IOFL) technique to preserve system robustness with respect to variations and uncertainties in stator and rotor resistances].

The experiments showed that this strategy demonstrated good performance. Abootorabi Zarchi [13] introduced a nonlinear speed tracking controller for a three-phase synchronous reluctance motor based on IOFL by considering the different control strategies related to this motor. The simulation and experimental results indicated that this approach was capable of decoupling control and exhibited good performance. Alonge [14] introduced the theoretical formulation of the IOFL control technique applied to linear IMs. The theory described the control 
design criteria by considering the constraints on the control and controlled variables that arose from applying this control technique in a real scenario. Afterward, they [15] described the set of tests in both the numerical simulations and the experiments, which presented achievable improvements in the dynamic performance of the control system.

The aforementioned methods have achieved good results to a certain extent. However, they are unable to realize dynamic decoupling and full linearization simultaneously. In addition, some of these methods have high computational complexity. Thus, the mathematical model for an IM system is first studied in the current research. Then, the NDG method is used for dynamic decoupling control and system linearization. Finally, pole assignment control techniques are applied to the linearization system during synthesis and simulation.

The remainder of this paper is organized as follows. Section 3 describes the mathematical model for an IM and its decoupling control technique based on NDG, along with the design of the controller. Section 4 presents several simulations to evaluate the performance of the proposed method. Conclusions are provided in Section 5.

\section{Methodology}

\subsection{Mathematical Model for the IM}

The mathematical model discussed in this paper is that for the AC IM powered by a voltage source in a two-phase arbitrary rotation $\mathrm{d}-\mathrm{q}$ coordinate system [16]. When the AC IM is powered by a voltage source-based inverter, the input control is a three-phase stator voltage. Therefore, the stator and the rotor are both converted into a two-phase arbitrary rotation $\mathrm{d}-\mathrm{q}$ coordinate system to facilitate analysis. In this manner, the dynamic mathematical model for the AC IM can be obtained. $x_{1}=i_{s d}$ and $x_{2}=i_{s q}$ are defined as the component of the stator current at the d- and q-axes, respectively; $x_{3}=\psi$ is the magnitude of the rotor flux; $x_{4}=\phi$ is the phase angle of the rotor flux relative to the daxis; $x_{5}=\omega_{r}$ is the rotor angular velocity; $u_{1}=u_{s d}$ and $u_{2}=u_{s q}$ are the component of the stator voltage at the dand q-axes, respectively; $u_{3}=\omega_{1}$ is the rotation angular velocity of the d-q coordinate system; $L_{s}$ and $L_{r}$ are the self-inductance of the stator and the rotor, respectively; $L_{m}$ is the mutual inductance between the stator and the rotor; $R_{1}$ and $R_{2}$ are the resistance of the stator and the rotor, respectively; is the number of pole pairs; and $\boldsymbol{J}$ is the moment of inertia of the system. The affine nonlinear form of the system model can be given as follows:

$$
\begin{aligned}
& x=f(x)+\sum_{j=1}^{3} g_{j}(x) \cdot u_{j} \\
& y_{i}=h_{i}(x), \quad i=1,2,
\end{aligned}
$$

Where, $x=\left[x_{1}, x_{2}, x_{3}, x_{4}, x_{5}\right]^{T}$

$$
\begin{aligned}
& f(x)=\left[\begin{array}{l}
-k_{1} x_{1}+k_{2} x_{3} \cos x_{4}+\frac{L_{m}}{\sigma L_{r}} x_{3} x_{5} \sin x_{4} \\
-k_{1} x_{2}+k_{2} x_{3} \sin x_{4}-\frac{L_{m}}{\sigma L_{r}} x_{3} x_{5} \cos x_{4} \\
\frac{L_{m}}{T_{r}}\left(x_{1} \cos x_{4}+x_{2} \sin x_{4}\right)-\frac{1}{T_{r}} x_{3} \\
\frac{L_{m}}{T_{r}} \frac{1}{x_{3}}\left(x_{2} \cos x_{4}-x_{1} \sin x_{4}\right)+x_{5} \\
k_{3} x_{3}\left(x_{2} \cos x_{4}-x_{1} \sin x_{4}\right)-\frac{n_{p}}{J} T_{l}
\end{array}\right] \\
& {\left[g_{1}(x) \quad g_{2}(x) \quad g_{3}(x)\right]=\left[\begin{array}{ccccc}
\frac{1}{\sigma} & 0 & 0 & 0 & 0 \\
0 & \frac{1}{\sigma} & 0 & 0 & 0 \\
x_{2} & -x_{1} & 0 & -1 & 0 \\
x^{2} & & &
\end{array}\right]^{T}}
\end{aligned}
$$

$$
\left[\begin{array}{lll}
h_{1}(x) & h_{2}(x) & h_{3}(x)
\end{array}\right]=\left[\begin{array}{lll}
x_{3} & x_{5} & x_{4}
\end{array}\right]
$$

Thus far, we have obtained an effective mathematical model for the AC IM, and the control method designed in the following sections is based on this model. Simultaneously, we also provide the following definitions, which are used in the subsequent sections:

$$
k_{1}=\frac{R_{1} L_{r}^{2}+R_{2} L_{m}^{2}}{\sigma L_{r}^{2}}, k_{2}=\frac{R_{2} L_{m}}{\sigma L_{r}^{2}}, k_{3}=\frac{n_{p}^{2} L_{m}}{J L_{r}}, T_{r}=\frac{L_{r}}{R_{2}},
$$

and

$$
\sigma=L_{s}-\frac{L_{m}^{2}}{L_{r}}
$$

\subsection{IM Control Based on NDG}

In this section, we describe how decoupling control and full linearization of the IM are realized based on the NDG method. The vector relationship degree is first calculated as follows.

\subsubsection{Calculating the Vector Relationship Degree}

The vector relationship degree is one of the most important basic concepts of NDG theory [17]. Consider a nonlinear single-input single-output system as follows:

$$
\begin{aligned}
& x=f(x)+g(x) u \\
& y=h(x)
\end{aligned} .
$$

If the following two conditions hold, then the relationship degree $r$ exists at point $x^{0}$.

(a) For all the $x$ in a neighborhood of $x^{0}$ and $k<r-1$, $L_{g} L_{f}^{k} h(x)=0$.

(b) $L_{g} L_{f}^{k-1} h\left(x^{0}\right) \neq 0$,

Where $L_{g} L_{f} h\left(x^{0}\right)$ is the LI derivative [18] operating on $h(x)$.

For the following MIMO system: 
$\left\{\begin{array}{l}x=f(x)+\sum_{i=1}^{m} g_{i}(x) u_{i} \\ y_{1}=h_{1}(x) \\ y_{m}=h_{m}(x)\end{array}\right.$,

Where $f(x)$ and $g_{i}(x)$ are the smooth vector fields, $h_{i}(x)$ is a smooth function defined in an open set of $R^{n}$, and the number of the input channels is equal to that of the output channels.

If the following two conditions hold, then the relationship degree $\left(r_{1}, r_{2}, \quad, r_{m}\right)$ exists at point $x^{0}$.

(a) For all the $x$ in a neighborhood of $x^{0}$ and $1 \leq i, j \leq m$, $k<r_{i}-1, L_{g} L_{f}^{k} h_{i}(x)=0$.

(b) The following $m \times m$ order matrix is nonsingular at point $x=x^{0}$ :

$$
A(x)=\left[\begin{array}{cc}
L_{g_{1}} L_{f}^{r_{1}-1} h_{1}(x) & L_{g_{m}} L_{f}^{r_{1}-1} h_{1}(x) \\
L_{g_{1}} L_{f}^{r_{2}-1} h_{2}(x) & L_{g_{m}} L_{f}^{r_{2}-1} h_{2}(x) \\
L_{g_{1}} L_{f}^{r_{m}-1} h_{m}(x) & L_{g_{m}} L_{f}^{r_{m}-1} h_{m}(x)
\end{array}\right]
$$

According to the definition of the introduced vector relationship degree, the following formulas can be obtained for the system model described in Eq. (1):

$$
\begin{aligned}
& {\left[\begin{array}{c}
\frac{\partial h_{1}}{x} \\
\frac{\partial h_{2}}{x} \\
\frac{\partial h_{3}}{x}
\end{array}\right]=\left[\begin{array}{lllll}
0 & 0 & 1 & 0 & 0 \\
0 & 0 & 0 & 0 & 1 \\
0 & 0 & 0 & 1 & 0
\end{array}\right]} \\
& L_{f} h_{1}(x)=\frac{\partial h_{1}(x)}{x} \cdot f(x)=\frac{L_{m}}{T_{r}}\left(x_{1} \cos x_{4}+x_{2} \sin x_{4}\right)-\frac{1}{T_{r}} x_{3} \\
& L_{f} h_{2}(x)=\frac{\partial h_{2}(x)}{x} \cdot f(x)=k_{3} x_{3}\left(x_{2} \cos x_{4}-x_{1} \sin x_{4}\right)-\frac{n_{p}}{J} T_{l} \\
& L_{f} h_{3}(x)=\frac{\partial h_{3}(x)}{x} \cdot f(x)=\frac{L_{m}}{T_{r}} \frac{1}{x_{3}}\left(x_{1} \cos x_{4}-x_{1} \sin x_{4}\right)+x_{5}
\end{aligned}
$$

For the output $h_{1}(x)$,

$$
\begin{aligned}
& {\left[\begin{array}{l}
L_{g_{1}} h_{1}(x) \\
L_{g_{2}} h_{1}(x) \\
L_{g_{3}} h_{1}(x)
\end{array}\right]=\left[\begin{array}{lll}
0 & 0 & 0
\end{array}\right]^{T}} \\
& L_{g_{1}} L_{f} h_{1}(x)=\frac{L_{m}}{\sigma T_{r}} \cos x_{4} \\
& L_{g_{2}} L_{f} h_{1}(x)=\frac{L_{m}}{\sigma T_{r}} \sin x_{4}
\end{aligned}
$$

$$
L_{g_{3}} L_{f} h_{1}(x)=0
$$

Thus, we obtain the vector relationship degree $r_{1}=2$.

For the output $h_{2}(x)$,

$$
\begin{aligned}
& {\left[\begin{array}{l}
L_{g_{1}} h_{2}(x) \\
L_{g_{2}} h_{2}(x) \\
L_{g_{3}} h_{2}(x)
\end{array}\right]=\left[\begin{array}{lll}
0 & 0 & 0
\end{array}\right]^{T}} \\
& L_{g_{1}} L_{f} h_{2}(x)=-\frac{k_{3} x_{3}}{\sigma} \sin x_{4}
\end{aligned}
$$

$$
L_{g_{2}} L_{f} h_{2}(x)=\frac{1}{\sigma} k_{3} x_{3} \cos x_{4}
$$

$$
L_{g_{3}} L_{f} h_{2}(x)=0
$$

Thus, we obtain the vector relationship degree $r_{2}=2$.

For the output $h_{3}(x)$,

$$
\left[\begin{array}{l}
L_{g_{1}} h_{3}(x) \\
L_{g_{2}} h_{3}(x) \\
L_{g_{3}} h_{3}(x)
\end{array}\right]=\left[\begin{array}{lll}
0 & 0 & -1
\end{array}\right]^{T}
$$

Thus, we obtain the vector relationship degree $r_{3}=1$. According to Eq. (8),

$$
\begin{aligned}
A(x) & =\left[\begin{array}{ccc}
L_{g_{1}} L_{f} h_{1}(x) & L_{g_{2}} L_{f} h_{1}(x) & L_{g_{3}} L_{f} h_{1}(x) \\
L_{g_{1}} L_{f} h_{2}(x) & L_{g_{2}} L_{f} h_{2}(x) & L_{g_{3}} L_{f} h_{2}(x) \\
L_{g_{1}} h_{3}(x) & L_{g_{2}} h_{3}(x) & L_{g_{3}} h_{3}(x)
\end{array}\right] \\
& =\left[\begin{array}{ccc}
\frac{L_{m}}{\sigma T_{r}} \cos x_{4} & \frac{L_{m}}{\sigma T_{r}} \sin x_{4} & 0 \\
-\frac{k_{3} x_{3}}{\sigma} \sin x_{4} & \frac{k_{3} x_{3}}{\sigma} \cos x_{4} & 0 \\
0 & 0 & -1
\end{array}\right]
\end{aligned}
$$

The determinant $\operatorname{det} A(x)=-2 \frac{K_{3} L_{m}}{\sigma^{2} T_{r}} x_{3}$ is nonsingular when $x_{3} \neq 0$, whereas it is singular when $x_{3}=0$; therefore, the overall relationship degree of the system is $r=r_{1}+r_{2}+r_{3}=5$. The dimension of the system is also 5 , which can ensure full linearization of the system.

\subsubsection{Decoupling Control and Full Linearization}

A coordinate transformation $z=\phi(x)$ is set as follows: 


$$
\left\{\begin{array}{l}
z_{11}=h_{1}(x)=x_{3} \\
z_{12}=L_{f} h_{1}(x)=\frac{L_{m}}{T_{r}}\left(x_{1} \cos x_{4}+x_{2} \sin x_{4}\right)-\frac{1}{T_{r}} x_{3} \\
z_{21}=h_{2}(x)=x_{5} \\
z_{22}=L_{f} h_{2}(x)=k_{3} x_{3}\left(x_{2} \cos x_{4}-x_{1} \sin x_{4}\right)-\frac{n_{p}}{J} T_{l} \\
z_{3}=h_{3}(x)=x_{4}
\end{array}\right.
$$

Then, we can obtain a transformed state equation in a new coordinate system as follows:

$$
\left\{\begin{array}{l}
z_{11}=z_{12} \\
z_{12}=L_{f^{2}} h_{1}(x)+L_{g_{1}} L_{f} h_{1}(x) u_{1}+L_{g_{2}} L_{f} h_{1}(x) u_{2}+L_{g_{3}} L_{f} h_{1}(x) u_{3} \\
z_{21}=z_{22} \\
z_{22}=L_{f^{2}} h_{2}(x)+L_{g_{1}} L_{f} h_{2}(x) u_{1}+L_{g_{2}} L_{f} h_{2}(x) u_{2}+L_{g_{3}} L_{f} h_{2}(x) u_{3} \\
z_{3}=L_{f} h_{3}(x)+L_{g_{1}} h_{3}(x) u_{1}+L_{g_{2}} h_{3}(x) u_{2}+L_{g_{3}} h_{3}(x) u_{3}
\end{array}\right.
$$

The control input is selected as

$$
u=A^{-1}(x)(-B(x)+v)
$$

Where $u=\left[u_{s d}, u_{s q}, \omega_{1}\right]^{T}$, and $v=\left[v_{1}, v_{2}, v_{3}\right]^{T}$ is the virtual input. $B(x)$ is

$$
\begin{aligned}
& B(x)=\left[\begin{array}{l}
b_{1}(x) \\
b_{2}(x) \\
b_{3}(x)
\end{array}\right]=\left[\begin{array}{lll}
L_{f^{2}} h_{1}(x) & L_{f^{2}} h_{2}(x) & L_{f^{2}} h_{3}(x)
\end{array}\right]^{T} \\
& {\left[-\frac{L_{m}}{T_{r}}\left(k_{1}+\frac{1}{T_{r}}\right) A_{1}(x)+\frac{1}{T_{r}}\left(k_{2} L_{m}+\frac{1}{T_{r}}\right) x_{3}+\frac{L_{m}^{2}}{T_{r}^{2} x_{3}} A_{2}^{2}(x)+\frac{L_{m}}{T_{r}} x_{2} A_{2}(x)\right]} \\
& =-k_{3}\left[\frac{L_{m}}{\sigma T_{r}} x_{3}^{2} x_{5}+x_{3} x_{5} A_{1}(x)+\left(k_{1}+\frac{1}{T_{r}}\right) x_{3} A_{2}(x)\right] \\
& \frac{L_{m}}{T_{r} x_{3}} A_{2}(x)+x_{5}
\end{aligned}
$$

Where

$$
\left\{\begin{array}{l}
A_{1}(x)=x_{1} \cos x_{4}+x_{2} \sin x_{4} \\
A_{2}(x)=x_{2} \cos x_{4}-x_{1} \sin x_{4}
\end{array}\right.
$$

Under the control operation in Eq. (25), the system in Eq. (24) can be divided into three independent subsystems. They are decoupled from one another, and no nonlinear component is present after linearization, which indicates that full linearization of the system is realized.

$$
\begin{aligned}
& {\left[\begin{array}{l}
z_{11} \\
z_{12}
\end{array}\right]=\left[\begin{array}{ll}
0 & 1 \\
0 & 0
\end{array}\right]\left[\begin{array}{l}
z_{11} \\
z_{12}
\end{array}\right]+\left[\begin{array}{l}
0 \\
1
\end{array}\right] v_{1}, y_{1}=\left[\begin{array}{ll}
1 & 0
\end{array}\right]\left[\begin{array}{l}
z_{11} \\
z_{12}
\end{array}\right]=\left\|\psi_{r}\right\|} \\
& {\left[\begin{array}{l}
z_{21} \\
z_{22}
\end{array}\right]=\left[\begin{array}{ll}
0 & 1 \\
0 & 0
\end{array}\right]\left[\begin{array}{l}
z_{21} \\
z_{22}
\end{array}\right]+\left[\begin{array}{l}
0 \\
1
\end{array}\right] v_{2}, y_{2}=\left[\begin{array}{ll}
1 & 0
\end{array}\right]\left[\begin{array}{l}
z_{21} \\
z_{22}
\end{array}\right]=\omega_{r}} \\
& z_{3}=v_{3}
\end{aligned}
$$

\subsection{System Controller Design}

Notably, Eq. (25) is not the only control law for the system. Moreover, it is also slightly complex. To make the control law easy to realize in practical applications, an appropriate simplification procedure should be performed.

\subsubsection{Control Law}

The general form of the control law is

$$
u=\alpha(x)+\beta(x) v=A^{-1}(x)[\xi(x)-b(x)+\Lambda(x) v]
$$

where

$$
\Lambda(x)=\left[\begin{array}{cc}
\lambda_{1}(x) & 0 \\
0 & \lambda_{m}(x)
\end{array}\right], \xi(x)=\left[\begin{array}{c}
\xi_{1}(x) \\
\xi_{m}(x)
\end{array}\right]
$$

$m$ is the demission of the system, which holds the following condition:

$$
\lambda_{i}(x), \xi_{i}(x) \in \operatorname{span}\left\{h_{i}(x), L_{f} h_{i}(x), \quad, L_{f^{\eta-1}} h_{i}(x)\right\}, i=1,2, \quad, n
$$

Evidently, the new control law given in Eq. (29) is significantly better than the old one; it can also be considerably simplified if $\Lambda(x)$ and $\xi(x)$ are selected appropriately. To enable the original system to still perform full linearization using the new control law, we set

$$
\begin{aligned}
& \lambda_{1}(x)=\lambda_{2}(x)=\lambda_{3}(x)=1 \\
& \xi(x)=\left[\begin{array}{l}
a_{11} h_{1}(x)+a_{12} L_{f} h_{1}(x) \\
a_{21} h_{2}(x)+a_{22} L_{f} h_{2}(x) \\
a_{3} h_{3}(x)
\end{array}\right]
\end{aligned}
$$

Simultaneously, we set

$$
a_{12}=a_{22}=-\left(k_{l}+\frac{1}{T_{r}}\right) \quad, \quad a_{11}=\frac{1}{T_{r}}\left(k_{2} L_{m}-k_{1}\right)
$$
, $a_{21}=a_{31}=0$.and; all of these values are constant. Then, we can obtain the control law $u(t)$ as follows:

$$
\begin{aligned}
& u(t)=\left[\begin{array}{l}
u_{s d} \\
u_{s q} \\
\omega_{l}
\end{array}\right]=A^{-1}(x)[\xi(x)-b(x)+\Lambda(x) v(t)] \\
& =\left[\begin{array}{ccc}
\frac{L_{m}}{\sigma T_{r}} \cos x_{4} & \frac{L_{m}}{\sigma T_{r}} \sin x_{4} & 0 \\
-\frac{k_{3} x_{3}}{\sigma} \sin x_{4} & \frac{k_{3} x_{3}}{\sigma} \cos x_{4} & 0 \\
0 & 0 & -1
\end{array}\right]\left\{\left[\begin{array}{l}
\frac{L_{m}^{2}}{T_{r}^{2} x_{3}} A_{2}^{2}(x)-\frac{L_{m}}{T_{r}} x_{2} A_{2}(x) \\
k_{3}\left[\frac{L_{m}}{\sigma T_{r}} x_{3}^{2} x_{5}+x_{3} x_{5} A_{1}(x)\right. \\
-\frac{L_{m}}{T_{r} x_{3}} A_{2}(x)+x_{5}
\end{array}\right]+\left[\begin{array}{l}
v_{1} \\
v_{2} \\
v_{3}
\end{array}\right]\right\}
\end{aligned}
$$

On the basis of the new control law given in Eq. (34), the system can be transformed into the following three decoupling subsystems after simplification, and full linearization can still be realized. 


$$
\begin{aligned}
& {\left[\begin{array}{l}
z_{11} \\
z_{12}
\end{array}\right]=\left[\begin{array}{cc}
0 & 1 \\
a_{11} & a_{22}
\end{array}\right]\left[\begin{array}{l}
z_{11} \\
z_{12}
\end{array}\right]+\left[\begin{array}{l}
0 \\
1
\end{array}\right] v_{1}} \\
& {\left[\begin{array}{l}
z_{21} \\
z_{22}
\end{array}\right]=\left[\begin{array}{cc}
0 & 1 \\
0 & a_{22}
\end{array}\right]\left[\begin{array}{l}
z_{21} \\
z_{22}
\end{array}\right]+\left[\begin{array}{l}
0 \\
1
\end{array}\right] v_{2}} \\
& z_{3}=v_{3}
\end{aligned}
$$

In addition, $z_{3}$ is the argument $\phi$, and the $\mathrm{d}-\mathrm{q}$ coordinate system rotates by the synchronous speed $\omega_{l}$ of the stator with respect to the three-phase coordinate system of the stationary stator; therefore, $z_{3}=\phi=0$. If we set $v_{3}=0$, then the system presented in Eq. (35) can be further simplified into two decoupling subsystems (i.e., the rotating speed and flux subsystems).

\subsubsection{Control Design}

We redesign reference inputs $v_{1}$ and $v_{2}$ to enable speed and flux to trace system expectations $\omega_{\text {ref }}$ and $\psi_{\text {ref }}$, as follows:

$\left\{\begin{array}{l}v_{1}=-K_{1}\left(z_{11}-\psi_{\text {ref }}\right)-K_{2} z_{12} \\ v_{2}=-K_{3}\left(z_{21}-\psi_{\text {ref }}\right)-K_{4} z_{22}\end{array}\right.$

As shown in Eq.

$\frac{d \psi_{r}}{d t}=z_{12}$ and $\frac{d z_{12}}{d t}=a_{11} \psi_{r}+a_{12} z_{12}+v_{1}$; hence, we can obtain

$\frac{d \psi_{r}^{2}}{d t^{2}}=a_{11} \psi_{r}+a_{12} \frac{d \psi_{r}}{d t}-K_{1}\left(\psi_{r}-\psi_{r e f}\right)-K_{2} \frac{d \psi_{r}}{d t}$

Then, the transfer function of the flux subsystem is

$$
G_{1}(s)=\frac{\psi_{r}(s)}{\psi_{r e f}(s)}=\frac{K_{1}}{s^{2}+\left(K_{2}-a_{12}\right) s+\left(K_{1}-a_{11}\right)}
$$

Similarly, $\frac{d \omega_{r}}{d t}=z_{21}$ and $\frac{d z_{21}}{d t}=a_{22} z_{21}+v_{2} ;$ therefore,

$\frac{d \omega_{r}^{2}}{d t^{2}}=a_{22} \frac{d \omega_{r}}{d t}-K_{3}\left(\omega_{r}-\omega_{r e f}\right)-K_{4} \frac{d \omega_{r}}{d t}$

The transfer function of the rotating speed subsystem is

$$
G_{2}(s)=\frac{\omega_{r}(s)}{\omega_{r e f}(s)}=\frac{K_{3}}{s^{2}+\left(K_{4}-a_{22}\right) s+K_{3}}
$$

Finally, we can obtain the closed-loop transfer function of the original system as follows:

$\left\{\begin{array}{l}G_{\left\|\psi_{r}\right\|}(s)=\frac{K_{1}}{s^{2}+\left(K_{2}-a_{12}\right) s+\left(K_{1}-a_{11}\right)} \\ G_{\omega_{r}}(s)=\frac{K_{3}}{s^{2}+\left(K_{4}-a_{22}\right) s+K_{3}}\end{array}\right.$

Where $\left(K_{1}, K_{2}\right)$ and $\left(K_{3}, K_{4}\right)$ are undetermined constants, which can enable the system to trace the input and output stably if they are selected appropriately.

Fig. 1 presents the structure diagram of the control system designed in this study. In the figure, the part within the dashed box shows the decoupling and linearization process of the nonlinear system, whereas the part outside the box shows the designed controller with input tracing and stable output achieved via the pole assignment method.

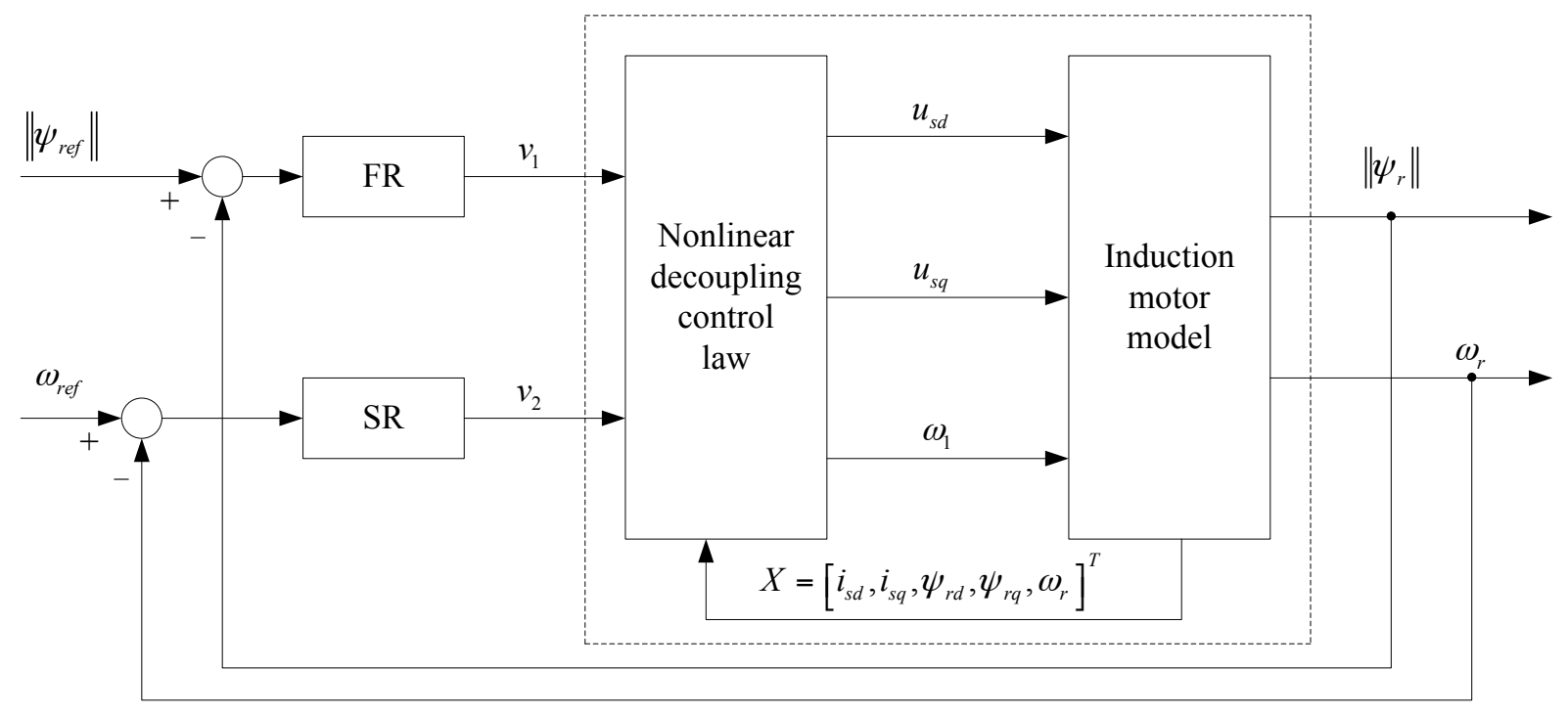

Fig.1. Structure diagram of the nonlinear decoupling control system for an IM.

\section{Results Analysis and Discussion}

In this section, two tests are presented to verify the effectiveness of the controller designed in this study. The simulation target is an AC IM that satisfies the system model presented in Eq. (1). The main parameters of this motor include the following: rated power $=2 \mathrm{KW}$, rated rotating speed $=185 \mathrm{rad} / \mathrm{s}, n_{p}=2, \mathrm{R} 1=0.685 \Omega, \mathrm{R} 2=0.847 \Omega, \mathrm{Ls}$ $=0.085 \mathrm{H}, \mathrm{Lr}=0.0863 \mathrm{H}, \mathrm{Lm}=0.0817 \mathrm{H}$, rated flux $=0.52$ $\mathrm{Wb}$, rated load $=13 \mathrm{Nm}$, and $\mathrm{J}=0.04 \mathrm{kgm} 2$. The definitions 
of all the symbols are the same as those in Section 3. For the designed closed-loop controller, the following conditions should be satisfied: overshoot $\sigma<5 \%$, peak ts $\leq 0.1 \mathrm{~s}$, and static error $e_{s p}=0$. Thus, we can calculate the undetermined constant $\left(K_{1}, K_{2}\right)=(1435,-165),\left(K_{3}, K_{4}\right)=(2503,-165)$.

In the first test, we set the rotating speed $\omega=120 \mathrm{rad} / \mathrm{s}$ and the flux $\psi=0.5 \mathrm{~Wb}$. The rotating speed $\omega$ will change from $120 \mathrm{rad} / \mathrm{s}$ step to $100 \mathrm{rad} / \mathrm{s}$ at $1.5 \mathrm{~s}$. We should determine how the flux will change during this process.

Figs. 2 and 3 show the response curve of the rotating speed and the flux, respectively. As shown in the figures, the rotating speed exhibits a fast response with a slight overshoot, which is within the acceptable range. The final output is stable at the set value. Simultaneously, the response of the flux is also fast and stable; moreover, it is unaffected by the step change in rotating speed.

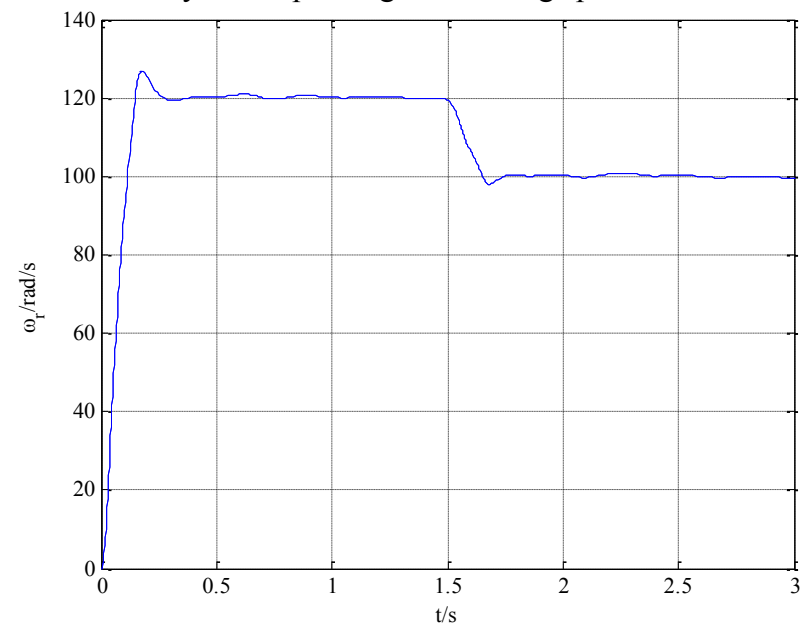

Fig. 2. Response curve of the rotating speed in test 1 .

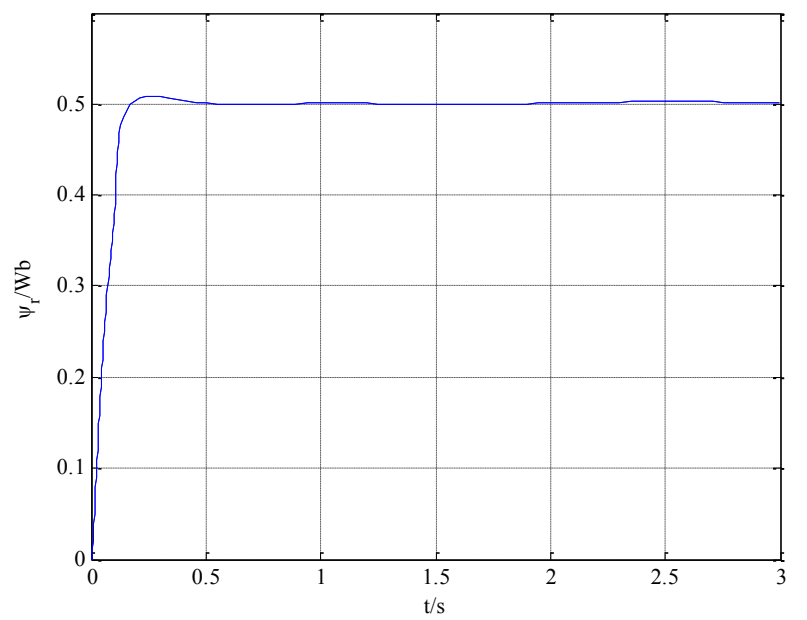

Fig. 3. Response curve of the flux in test 1.

In the second test, we set the flux change from $0.5 \mathrm{~Wb}$ step to $0.4 \mathrm{~Wb}$ at $2.0 \mathrm{~s}$ and then observe how rotating speed will change during this process.

Similar to test 1, Fig. 4 and 5 shows that during the mutation process, the response of the flux is extremely fast, the overshoot is within the acceptable range, and the steadystate error is nearly equal to zero. Meanwhile, the rotating speed of the motor does not significantly change and is substantially maintained at $120 \mathrm{rad} / \mathrm{s}$, which demonstrates good robustness.

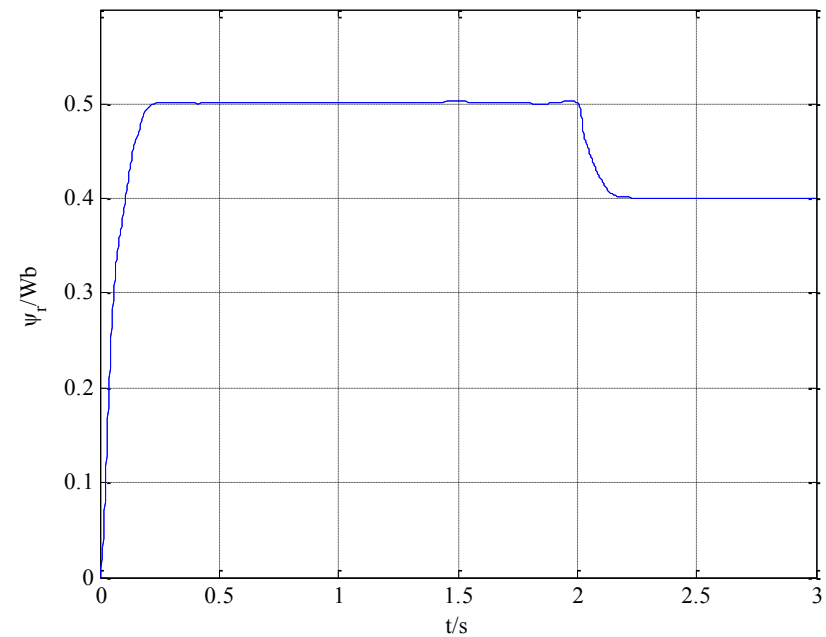

Fig. 4. Response curve of the flux in test 2.

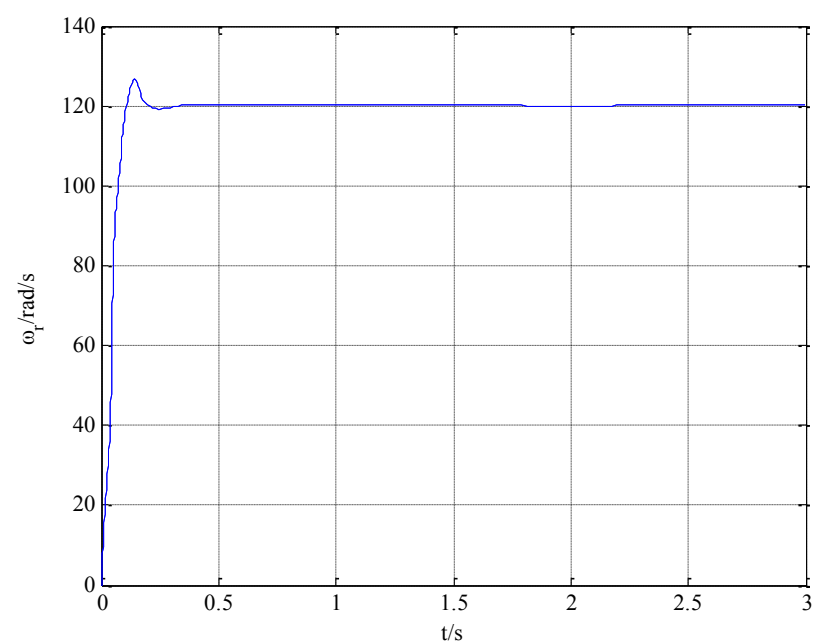

Fig. 5. Response curve of the rotating speed in test 2.

In summary, the controller based on the NDG method designed in this study can realize decoupling and full linearization for an AC IM. The motor model can be decoupled into two independent subsystems and exhibits good steady and dynamic performances.

\section{Conclusions}

This study proposes a new control strategy to decouple an AC IM based on the NDG approach. The vector relationship degree is used to determine whether the model can achieve full linearization. Meanwhile, the pole assignment method is used to design the control law and the controller. Several simulations are conducted to evaluate the performance of the proposed decoupling control method for an IM. The main conclusions of this study are as follows.

(1)A five-order affine mathematical model for an IM is established. This model can effectively describe and reflect the characteristic of an IM and achieve good balance between model accuracy and complexity.

(2)By calculating the vector relationship degree of the model presented in Section 3.1, we determine that the vector relationship degree is equal to the dimension of the system. This finding indicates that the established model can achieve full linearization. Therefore, we use a simple coordinate transformation to realize system decoupling. 
(3)A general and simple control law is established, and a controller is designed based on this law using the pole assignment method. The simulation results show that the proposed method exhibits good dynamic and static performances. The induction model can be decoupled into two independent subsystems, and a change in one subsystem does not affect the other subsystem. Meanwhile, both response time and overshoot satisfy the requirement.

Nevertheless, the proposed method requires an accuracy system model and several important parameters should be known in advance. Such condition is difficult to satisfy in practical applications. In the future, we will attempt to combine this method with an adaptive parameter identification technique to solve the problem of model and parameter uncertainty.

\section{Acknowledgements}

This work was supported by the National Natural Science Foundation of China (Grant No.11426158) and by the Capital University of Economics and Business (Grant No. 2014XJQ011).

\section{References}

1. Tomasz Szolc, Robert Konowrocki, Maciej Michajłow, et al., “An investigation of the dynamic electromechanical coupling effects in machine drive systems driven by asynchronous motors", Mechanical Systems and Signal Processing, 49(1-2), 2014, pp.118134.

2. Cemal Yilmaz, Osman Gvrdal, Ilhan Kosalay, "Network induced delay of asynchronous motor connected to Profibus-DP networks using fuzzy logic control algorithm", Expert Systems with Applications, 37(4), 2010, pp. 3248-3255.

3. Shaowei Wang, Shanming Wan, "Adaptive velocity control in AC asynchronous motor based on identification of load parameters by VMACO", Procedia Engineering, 15(8), 2011, pp. 45-49.

4. Yuksel Oguz, Mehmet Dede, "Speed estimation of vector controlled squirrel cage asynchronous motor with artificial neural networks", Energy Conversion and Management, 52(1), 2011, pp. 675-686.

5. Ravi Gondhalekar, Colin N. Jones, "MPC of constrained discretetime linear periodic systems-A framework for asynchronous control: Strong feasibility, stability and optimality via periodic invariance", Automatica, 47(2), 2011, pp. 326-333.

6. N. Bounar, A.Boulkroune, F.Boudjema, et al., "Adaptive fuzzy vector control for a doubly-fed induction motor", Neurocomputing, 151(3), 2015, pp. 756-769.

7. Zheng-Qi WANG, Xian-Xing LIU, "Nonlinear Internal Model Control for Bearingless Induction Motor Based on Neural Network Inversion", Acta Automatica Sinica, 39(4), 2013, pp. 433-439.

8. Ramdane Hedjar, Patrick Boucher, Didier Dumur, "Robust nonlinear receding-horizon control of induction motors", Electrical Power and Energy Systems, 46(3), 2013, pp. 353-365.

9. Liao Zhiling, Jia Hongping, Liu Guohai,"Comparative Study on Vector Control and Differential Geometry Decoupling Control Method of Induction Motor", 2005 International Conference on Electrical Machines and Systems, Nanjing, China: IEEE, 2005, pp. 1539-1543.
10. Zhaojun Meng, Changzhi Sun, Yuejun An, et al., "Nonlinear decoupling control of induction motor based on parameter adaptive identification", 2011 International Conference on Intelligent Human-Machine Systems and Cybernetics, Zhejiang, China: IEEE, 2011, pp. 98-102.

11. Cao Li, Feng Dongmei, Diao Xiaoyan, et al., "The Decoupling Control of Bearingless Synchronous Reluctance Motor Based on Differential Geometry", 2012 Chinese Control Conference, Hefei, China: IEEE, 2012, pp. 4261-4266.

12. R. Yazdanpanah, J. Soltani, G.R. Arab Markadeh, "Nonlinear torque and stator flux controller for induction motor drive based on adaptive input - output feedback linearization and sliding mode control", Energy Conversion and Management, 49(4), 2008, pp. $541-550$.

13. H. Abootorabi Zarchi, Gh.R. Arab Markadeh, J. Soltani, "Direct torque and flux regulation of synchronous reluctance motor drives based on input - output feedback linearization", Energy Conversion and Management, 51(1), 2010, pp. 71-80.

14. Francesco Alonge, Maurizio Cirrincione, Marcello Pucci, et al., "Input-output feedback linearizing control of linear induction motor taking into consideration the end-effects. Part I: Theoretical analysis", Control Engineering Practice, 36(3), 2015, pp. 133-141.

15. Francesco Alonge, Maurizio Cirrincione, Marcello Pucci, et al., "Input-output feedback linearizing control of linear induction motor taking into consideration the end-effects. Part II: Simulation and experimental results", Control Engineering Practice, 36(3), 2015, pp. 142-150.

16. Luis Amezquita-Brooks, Eduardo Liceaga-Castro, Jesus LiceagaCastro, "Novel design model for the stator currents subsystem of induction motors", Applied Mathematical Modelling, 38(23), 2014, pp. 5623-5634.

17. George Shapiro, "On discrete differential geometry in twistor space", Journal of Geometry and Physics, 68(6), 2013, pp. 81-102.

18. Arash Yazdanbakhsh, Simone Gori, "Mathematical analysis of the Accordion Grating illusion: A differential geometry approach to introduce the 3D aperture problem", Neural Networks, 24(10), 2011, pp. 1093-1101. 